\title{
Effect of poly- $\alpha, \gamma$, L-glutamic acid as a capping agent on morphology and oxidative stress-dependent toxicity of silver nanoparticles
}

This article was published in the following Dove Press journal:

International Journal of Nanomedicine

I I November 201।

Number of times this article has been viewed

\section{Magdalena Stevanović \\ Branimir Kovačević ${ }^{2}$ \\ Jana Petkovićs \\ Metka Filipič ${ }^{3}$ \\ Dragan Uskokovićl}

'Institute of Technical Sciences of Serbian Academy of Sciences and Arts, ${ }^{2}$ Institute of General and Physical Chemistry, Belgrade, Serbia; ${ }^{3}$ Department of Genetic Toxicology and Cancer Biology, National Institute of Biology, Ljubljana, Slovenia
Correspondence: Magdalena Stevanović Institute of Technical Sciences of Serbian Academy of Sciences and Arts, Knez Mihailova 35/IV, I 1000 Belgrade, Serbia Tel +38 II I 2636994

$\mathrm{Fax}+38$ II 12185263

Email magdalena.stevanovic@itn.sanu.ac.rs

\begin{abstract}
Highly stable dispersions of nanosized silver particles were synthesized using a straightforward, cost-effective, and ecofriendly method. Nontoxic glucose was utilized as a reducing agent and poly- $\alpha, \gamma$, L-glutamic acid (PGA), a naturally occurring anionic polymer, was used as a capping agent to protect the silver nanoparticles from agglomeration and render them biocompatible. Use of ammonia during synthesis was avoided. Our study clearly demonstrates how the concentration of the capping agent plays a major role in determining the dimensions, morphology, and stability, as well as toxicity of a silver colloidal solution. Hence, proper optimization is necessary to develop silver colloids of narrow size distribution. The samples were characterized by Fourier transform infrared spectroscopy, ultraviolet-visible spectroscopy, field-emission scanning electron microscopy, transmission electron microscopy, and zeta potential measurement. MTT assay results indicated good biocompatibility of the PGAcapped silver nanoparticles. Formation of intracellular reactive oxygen species was measured spectrophotometrically using 2,7-dichlorofluorescein diacetate as a fluorescent probe, and it was shown that the PGA-capped silver nanoparticles did not induce intracellular formation of reactive oxygen species.
\end{abstract}

Keywords: silver nanoparticles, poly- $\alpha, \gamma$, L-glutamic, green synthesis, morphology, cytotoxicity

\section{Introduction}

In recent years, nanoscience has increased and expanded from a multidisciplinary research concept to a primary scientific frontier. Rapid technological advancements have led to the expansion of nanoscale device components, advanced sensors, and novel biomimetic materials. ${ }^{1,2}$ Among the noble metals, silver nanoparticles have received considerable attention due to their attractive physicochemical properties, such as magnetic and optical polarizability, electrical conductivity, catalysis, antimicrobial behavior, DNA sequencing, surface-enhanced Raman scattering, and thermal properties. ${ }^{3,4}$ Silver nanoparticles have been found to have strong antimicrobial activity, and are used in wound dressings, contraceptive devices, surgical instruments, bone prostheses, and as coating for ocular lenses to prevent microbial activity. ${ }^{2,5,6}$ However, the potentially negative impacts of nanomaterials are sometimes overlooked during the discovery phase of research. The implementation of green chemistry principles can enhance nanoscience by maximizing safety and efficiency, while minimizing the environmental and societal impacts of these materials. ${ }^{1}$

The general principle of synthesis of metal nanoparticles from a salt solution is based on using a reducing agent in the presence of a capping agent. Commonly used 
reductants include borohydride, citrate, ascorbate, elemental hydrogen, tetrabutylammonium borohydride, organometallic compounds, dimethylformamide, potassium bitartrate, alcohols, and polyols. ${ }^{7-13}$ Capping agents prevent nanoparticles from agglomerating, as well as modifying their morphology. Polyvinyl pyrrolidone, polyvinyl alcohol, gelatin, carboxymethylcellulose, polyacrylnitrile, starch, gelatin, heparin, chitosan, bovine serum albumin, polysaccharides, and oleylamine are the most often used capping agents for silver nanoparticles. ${ }^{14-18}$ A problem which can occur with the particle stabilizer is the difficulty of removing it from the system, and this is addressed in the literature. ${ }^{19}$ The remains of the stabilizer often modify the surface characteristics of the particles, thus affecting biocompatibility and distribution throughout the body. ${ }^{19}$ Therefore, it is necessary to determine accurately the concentration of stabilizers for any given method.

Herein we demonstrate, for the first time, a simple and quick method of synthesis of uniform and stable silver nanoparticles, using nontoxic glucose as the reduction agent, poly- $\alpha, \gamma$, L-glutamic acid (PGA) as the capping agent, and without using ammonia. A number of researchers have successfully synthesized silver nanoparticles, but use of this green, one-pot, four-component method overcomes some of the disadvantages previously reported using other methods, such as impurities, solvent toxicity, difficulty in controlling particle size and distribution, economic viability, and problems in preparation that limit their commercialization potential. $^{12,15,18,20}$ The major advantage of this method of synthesis of silver nanoparticles, relative to other methods, is most certainly use of PGA as the capping agent. PGA is a nontoxic, hydrophilic, and biodegradable polymer utilized to enhance the stability and cytocompatibility of silver nanoparticles. PGA is a naturally occurring, multifunctional, biodegradable biopolymer of L-glutamic acid produced by fermentation with Bacillus subtilis, ${ }^{21}$ and has been developed rapidly in the last two decades. ${ }^{21}$ Incorporation of PGA into an antigenic formulation, influenza vaccine, and gene transfection carrier has led to improved pharmaceutical efficacy. ${ }^{21}$

The use of PGA as a capping agent has already been reported by another group, ${ }^{22}$ but with use of ammonia in the synthesis, which was avoided using our method. Ammonia is a very corrosive and hazardous chemical, so is avoided in a number of other reported synthesis procedures, with use of alternative chemicals as reducing and/or capping agents, eg, sodium borohydride, dimethyl formamide, and cetyltrimethylammonium bromide. ${ }^{7-9,13,23,24}$ However, there is a general concern about these products because of their potential environmental and biological risks. These studies have also contributed to our understanding of the role played by a capping agent in size, morphology, and stability control. To address this issue, we have done a series of experiments under identical conditions (ie, precursor, temperature, reductant, concentrations of reagents, and reaction time) except for the use of different concentrations of capping agent. We found that particles of varying shape, size, uniformity, agglomeration, and stability could be produced by adding different amounts of capping agent.

The environmental and health impact of nanomaterials is becoming an important topic in research over recent years. The unique advantages offered by these nanomaterials in a wide range of applications cannot be realized until these concerns are resolved. ${ }^{25}$ As shown by toxicokinetic studies, the liver is expected to be the major organ for systemic toxicity of silver nanoparticles. ${ }^{26}$ Therefore, in our study, we used a test system of human HepG2 hepatoma cells in order to evaluate the in vitro cytotoxic potential of bare and PGAcapped silver nanoparticles using the 3-(4,5-dimethylthiazol-2-yl)-2,5-diphenyltetrazolium bromide (MTT) assay. Recent studies have pointed to a higher toxic potential using nanomaterials than their larger counterparts, probably due to oxidative stress as a consequence of increased production of reactive oxygen species. ${ }^{27,28}$ In this study, we also determined whether bare and PGA-capped silver nanoparticles could cause increased production of reactive oxygen species.

\section{Materials and methods Materials}

Silver nitrate $\left(\mathrm{AgNO}_{3}\right.$, molar ratio 169.88) was obtained from Centrohem (Stara Pazova, Serbia), glucose $\left(\mathrm{C}_{6} \mathrm{H}_{12} \mathrm{O}_{6}\right.$, molar ratio 180.20) from Zorka Pharma (Sabac, Serbia), and sodium hydroxide ( $\mathrm{NaOH}$, molar ratio 40.00) from Kemika (Zagreb, Croatia). PGA with a molecular weight of $20-40 \mathrm{kDa}(99.9 \%$ high-pressure liquid chromatography purity) was purchased from Guilin Peptide Technology Limited (Guangxi, China). All reagents were of analytic grade and used as received without further purification.

Chemicals, ie, Eagle's minimal essential medium, penicillin/streptomycin, L-glutamine, phosphate-buffered saline, trypsin, fetal bovine serum, nonessential amino acid solution $(100 \times)$, MTT, dimethyl sulfoxide, tert-butyl hydroperoxide $(t-\mathrm{BOOH})$ and 2,7-dichlorofluorescein diacetate (DCFH-DA) used for determining cytotoxicity and formation of reactive oxygen species were obtained from Sigma-Aldrich (St Louis, MO). 


\section{Methods}

\section{Green synthesis of bare and PGA-capped silver nanoparticles}

The route to synthesis of silver nanoparticles with and without PGA as the capping layer and without use of ammonia is new, simple, and completely green. Bare and PGA-capped silver nanoparticles were synthesized using a chemical reduction method whereby silver nitrate, glucose, and PGA served as a precursor, a reduction agent, and a capping agent, respectively.

The initial solution used in all syntheses was $20 \mathrm{~mL}$ of distilled water into which $0.5 \mathrm{~mL}(0.5 \mathrm{wt} \%) \mathrm{NaOH}, 10 \mathrm{~mL}$ ( $1 \mathrm{wt} \%)$ glucose, and $0.5 \mathrm{~mL}(0.1 \mathrm{wt} \%) \mathrm{AgNO}_{3}$ were introduced, with continuous stirring on a magnetic stirrer at $300 \mathrm{rpm}$. Glucose in an alkaline solution is oxidized by oxygen, forming gluconic acid, which is converted to sodium gluconate, a noncorrosive and nontoxic compound used as a food additive or fortifier of sodium in the presence of sodium hydroxide. Silver nitrate speeds up the reaction by acting as an oxidizing agent. As glucose is oxidized by dissolved oxygen, silver ions are reduced, forming silver nanoparticles, and the solution takes on a yellow-green color. For the formation of the capped silver nanoparticles, $0.5 \mathrm{~mL}$ of $0.05 \mathrm{wt} \%$ (0.05), $0.1 \mathrm{wt} \%(0.1), 0.2 \mathrm{wt} \%(0.2), 0.4 \mathrm{wt} \%$ (0.4), or $0.5 \mathrm{wt} \%$ (0.5) PGA was added to the solution before adding $\mathrm{AgNO}_{3}$. The preparation was completed in a single reaction vessel to facilitate fabrication.

\section{Ultraviolet spectroscopy}

Ultraviolet measurements were performed using a GBC Cintra ultraviolet-visible spectrophotometer at a frequency interval of 200-600 nm. Ultraviolet-visible spectroscopy was used to estimate the extent of formation of silver nanoparticles during the reaction, the formation of PGA-capped silver nanoparticles, the stability of the silver nanoparticles over a given time period, and the extent of formation of silver nanoparticles using different amounts of capping agent, ie, the influence of capping agent concentration on the final products.

\section{Fourier-transform infrared spectroscopy}

Analysis of the quality of the samples was performed using Fourier transform infrared (FTIR) spectroscopy. FTIR spectra for the samples were recorded in the range of $400-4000 \mathrm{~cm}^{-1}$ using a Specord 75 spectrometer (Carl Zeiss, Jena, Germany) at $4 \mathrm{~cm}^{-1}$ resolution. FTIR measurements of the samples were carried out to identify possible interactions between silver and PGA which might be responsible for stabilization, ie, protection of the silver nanoparticles from agglomeration and rendering them biocompatible.

\section{Field emission scanning electron microscopy}

Observation of the microstructure of the bare and capped silver nanoparticles using different amounts of PGA as the capping agent was done using field-emission scanning electron microscopy (FESEM). FESEM measurements were performed on a Supra 35 VP field-emission scanning electron microscope (Carl Zeiss). The samples were prepared by redispersion in ethanol using an ultrasonic bath and filtering the dispersions using polycarbonate membranes. Carbon coating was used to prevent charging.

\section{Transmission electron microscopy}

Transmission electron microscopy (TEM) with a JEM-2100 (JEOL, Peabody, MA) was employed for further morphological characterization of the bare and PGA-capped silver nanoparticles by exploring the individual nanostructures. Samples for the TEM analysis were prepared by dispersing the powders in distilled water using an ultrasonic bath. The suspensions were subsequently dropped on a lacey carbon film supported by a 300-mesh copper grid.

\section{Zeta potential measurement}

The zeta potential was measured using a Zetasizer (Nano ZS, Model ZEN3600, Malvern Instruments, Worcestershire, UK), with a particle size range for zeta potential determination of 5-10 $\mu \mathrm{m}$, using the principle of electrophoretic mobility under an electric field. The zeta potential is a function of the dispersion/suspension $\mathrm{pH}$ which determines particle stability in dispersion.

\section{Cell culture}

HepG2 cells were obtained from the European Collection of Cell Cultures. The cells were grown in Eagle's minimal essential medium containing $10 \%$ fetal bovine serum, $1 \%$ nonessential amino acid solution, $2 \mathrm{mM}$ L-glutamine, and $100 \mathrm{U} / \mathrm{mL}$ penicillin plus $100 \mu \mathrm{g} / \mathrm{mL}$ streptomycin at $37^{\circ} \mathrm{C}$ in a humidified atmosphere and $5 \% \mathrm{CO}_{2}$.

\section{Cytotoxicity of bare and PGA-capped silver nanoparticles}

The cytotoxicity of the bare and PGA-capped silver nanoparticles was determined using the MTT assay according to Mosmann, ${ }^{29}$ with minor modifications. ${ }^{30}$ This assay measures the conversion of MTT to insoluble formazan 
by dehydrogenase enzymes from the intact mitochondria of living cells.

HepG2 cells were seeded into 96-well microplates (Nunc, Naperville, IL) at a density of 40,000 cells $/ \mathrm{mL}$ and incubated for 20 hours at $37^{\circ} \mathrm{C}$ for attachment. The medium was then replaced by fresh complete medium containing $0 \%, 0.01 \%, 0.1 \%, 1 \%$, and $10 \% \mathrm{v} / \mathrm{v}$ of bare and PGAcapped silver nanoparticles, and incubated for 24 hours. In each experiment, a negative control (nontreated cells) and vehicle control (10\% emulsion) was included. MTT (final concentration $0.5 \mathrm{mg} / \mathrm{mL}$ ) was then added, and incubated for a further 3 hours. In the next step, the medium and MTT was removed, and the formazan crystals formed were dissolved in dimethylsulfoxide. The optical density was measured at $570 \mathrm{~nm}$ (reference filter $690 \mathrm{~nm}$ ) using a microplate-reading spectrofluorometer (Tecan Genios, MTX Lab Systems Inc, Vienna, VA). Survival (viability) was determined by comparing the optical density of the wells containing the treated cells with those of the nontreated cells. Five replicates per concentration point and three independent experiments were performed.

The results are expressed as the mean values of three independent experiments. One-way analysis of variance (Kruskal-Wallis) was used for evaluation of the statistical significance of any differences between the exposed and control cells and between cells exposed to bare and PGAcapped silver nanoparticles. $P<0.05$ was considered to be statistically significant.

\section{Intracellular reactive oxygen species formation}

Formation of intracellular reactive oxygen species was measured spectrophotometrically using a fluorescent probe, ie, DCFH-DA, as described by Osseni et al, ${ }^{31}$ with minor modifications. ${ }^{32}$ DCFH-DA readily diffuses through the cell membrane and is hydrolyzed by intracellular esterases to nonfluorescent $2^{\prime}, 7^{\prime}$-dichlorofluorescein. It is then rapidly oxidized to highly fluorescent $2^{\prime}, 7^{\prime}$ - DCFH-DA in the presence of reactive oxygen species. The fluorescence intensity is proportional to the amount of intracellular reactive oxygen species formed.

HepG2 cells were seeded at a density of 75,000 cells $/ \mathrm{mL}$ in 96-well, black, tissue culture-treated microtiter plates in five replicates. After 20 hours of incubation at $37^{\circ} \mathrm{C}$ in $5 \% \mathrm{CO}_{2}$, the cells were incubated with $20 \mu \mathrm{M}$ DCFH-DA. After 30 minutes, DCFH-DA was removed and the cells were treated with $0 \%, 0.01 \%, 0.1 \%, 1 \%$, and $10 \% \mathrm{v} / \mathrm{v}$ of the bare and PGA-capped silver nanoparticles in phosphatebuffered saline. Negative (nontreated cells), vehicle (10\% emulsion), and positive control (0.5 $\mathrm{mM} t$-BOOH) groups were included in each experiment. For the kinetic analyses, the dishes were maintained at $37^{\circ} \mathrm{C}$ and the fluorescence intensity was determined every 30 minutes during a 5-hour incubation using the microplate-reading spectrofluorometer at an excitation wavelength of $485 \mathrm{~nm}$ and an emission wavelength of $530 \mathrm{~nm}$.

Statistically significant differences between the treated groups and controls, and between cells treated with bare and PGA-capped silver nanoparticles were determined by one-way analysis of variance (Kruskal-Wallis) with Dunnett's test and Bonferroni's post-test, respectively. $P<0.05$ was considered to be statistically significant.

\section{Results and discussion Ultraviolet-visible spectroscopy}

The ultraviolet-visible absorption results confirmed formation of bare and PGA-capped silver nanoparticles prepared by new, simple, green, one-pot four-component synthesis (Figure 1). The successful synthesis of bare and PGA-capped silver nanoparticles was first indicated by the distinctive color that appeared in the colloidal solution. Initially, reduction of silver ions leads to formation of silver atoms, followed by agglomeration into clusters. ${ }^{6}$ These clusters eventually lead to formation of colloidal silver particles. ${ }^{6}$ When the colloidal particles are much smaller than the wavelength of visible light, the solutions develop a yellow color with an intense band around $400 \mathrm{~nm} .{ }^{33}$ This band is attributable to collective excitation of the electron gas in the particles, with a periodic change in electron density at the surface (surface plasmon absorption). ${ }^{33,34}$

Silver nanoparticles are especially valued for their optical properties, being useful for surface-enhanced Raman scattering, imaging, sensing, and wave guiding applications, where the optical absorption arising from the surface plasmons of noble metal materials is important. The position and shape of the surface plasmon absorption band is strongly dependent on the size and shape of the particle, the dielectric constant of the medium, and surface-adsorbed species. $^{33-35}$

The ultraviolet-visible spectra for bare silver nanoparticles shows only one dominant, broad absorption band around $421 \mathrm{~nm}$, which represents the typical signature of the dipolar plasmon resonance of silver nanoparticles. ${ }^{33-35}$ The ultraviolet-visible absorption spectra of the silver nanoparticles capped with different concentrations of PGA were also investigated in our study. Ultraviolet-visible absorption spectra for the PGA (0.05)-capped silver nanoparticles and PGA (0.1)-capped silver nanoparticles showed 
A

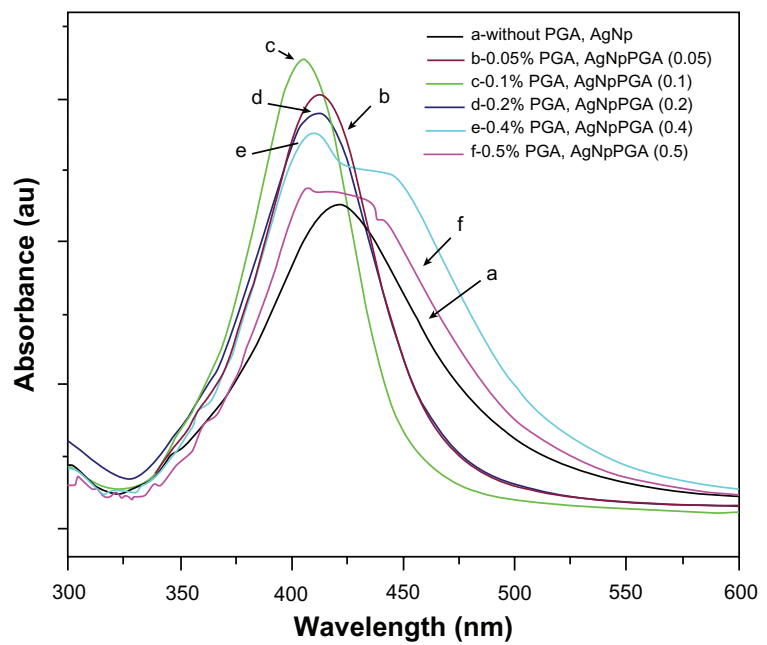

C

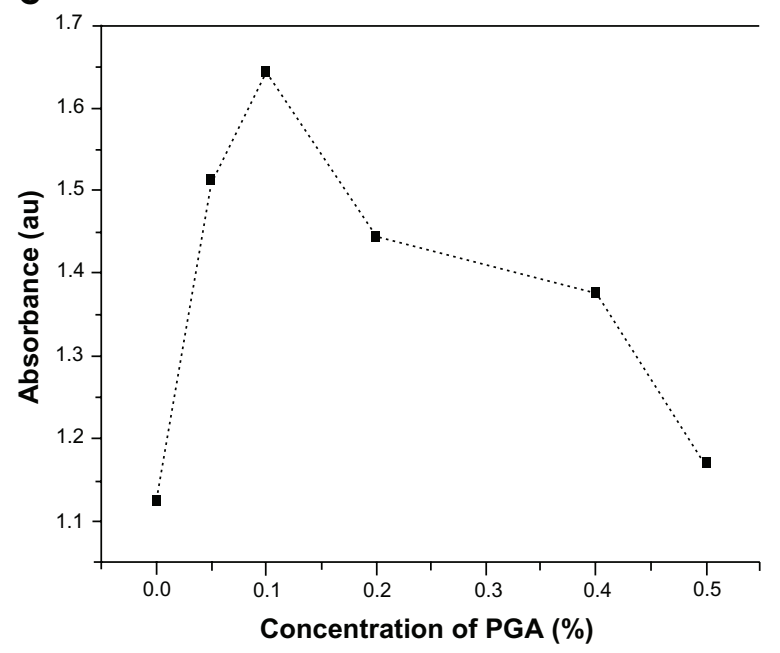

B

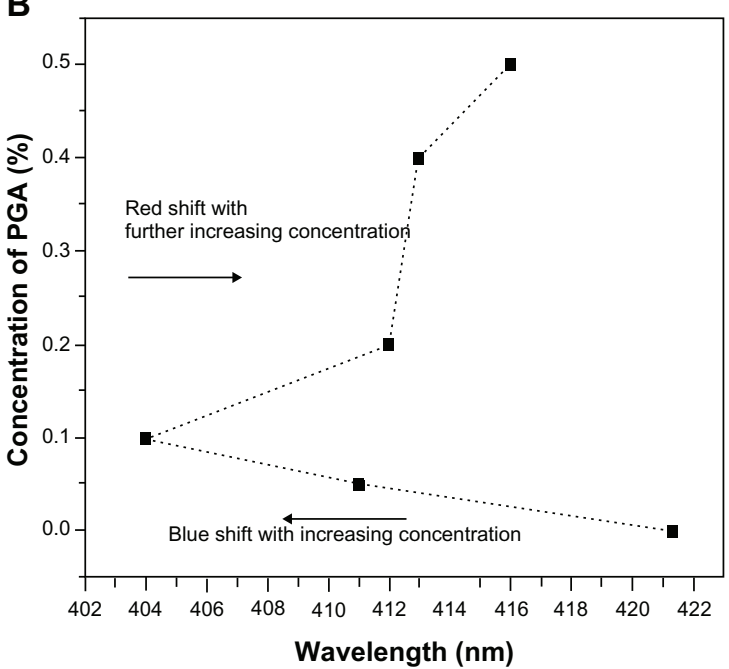

D

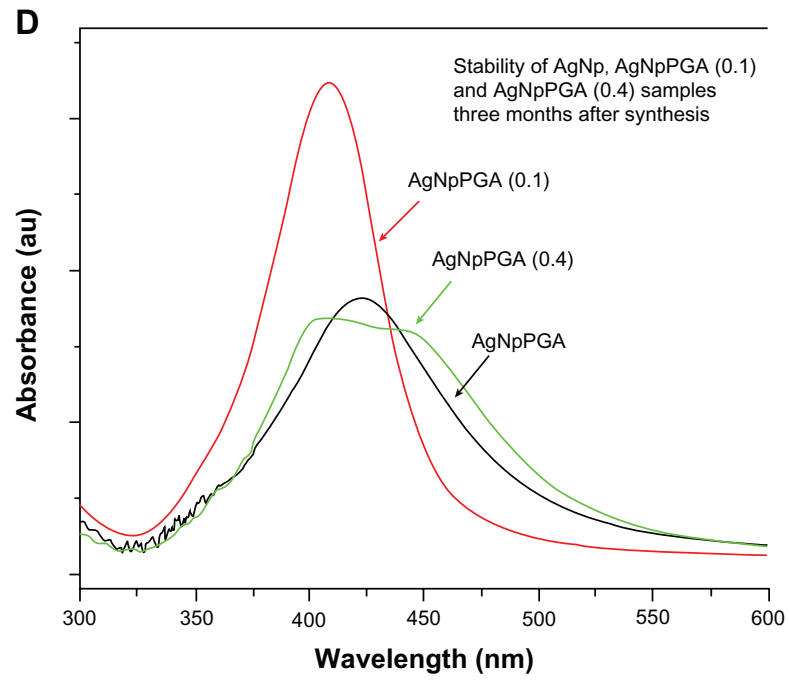

Figure I (A) UV-visible spectra of bare silver nanoparticles and capped with different amounts of PGA as capping agent, (B) Absorbance maximum displacement depending on the concentration of capping agent; (C) Increase of the absorbance maximum depending on the concentration of capping agent and (D) Stability of bare and PGA capped silver nanoparticles three months after synthesis.

Abbreviations: PGA, poly- $\alpha, \gamma$, L-glutamic acid; AgNpPGA, PGA-capped silver nanoparticles.

narrow surface plasmon absorption bands at $411 \mathrm{~nm}$ and at $404 \mathrm{~nm}$ wavelengths, respectively. The maximum absorption displacement for the PGA (0.05)-capped silver nanoparticles as well as for the PGA (0.1)-capped silver nanoparticles at the smaller wavelengths (blue shift) indicates a change in particle size to smaller dimensions (Figure 1B). It is well known from the literature that a polaritonic blue shift occurs with decreasing cluster size and a red shift occurs with increasing cluster size, due to electromagnetic retardation. ${ }^{33}$ Shifting of the absorption band to shorter wavelengths also indicates that the particles became more spherical with the addition of PGA. Although it was expected that this trend would continue with further increases in PGA concentration, Figure 1 shows that this did not happen for the PGA (0.2), (0.4) or (0.5)-capped silver nanoparticles. With increases in PGA concentration beyond $0.1 \%$, the absorption spectra become broader, with a decrease in absorbance, and also showed red-shifted peaks at $412 \mathrm{~nm}, 413 \mathrm{~nm}$, and $416 \mathrm{~nm}$ for PGA (0.02), (0.4) and (0.5)-capped silver nanoparticles, respectively (Figure $1 \mathrm{~A}, \mathrm{~B}$, and $\mathrm{C}$ ). It was observed that the absorption spectrum for PGA (0.4)-capped silver nanoparticles became very broad, with a decrease in absorbance at $413 \mathrm{~nm}$ and a shoulder appearing around $485 \mathrm{~nm}$. Resonances of higher multipolar order appeared in addition to the usual dipolar resonance when the cluster size increased, so the optical field became nonuniform across the cluster. In clusters of nonspherical shape, the single dipolar resonance of the spherical case splits into two or more nondegenerate plasmon modes that differ in their oscillation directions. ${ }^{33,34}$ All these observations indicate that the optimum concentration of PGA for formation of PGA-capped silver nanoparticles is $0.1 \%$. 
The stability of nanoparticle dispersions is a key factor in their successful application. In order to prevent agglomeration of the nanoparticles, different concentrations of capping agent were added to the reaction media. The absorption spectra of solutions containing PGA (0.1)-capped silver nanoparticles, recorded immediately after preparation and after three months of storage, confirmed very good stability of the samples as opposed to samples containing bare silver nanoparticles or silver nanoparticles capped with more than $0.1 \%$ PGA (Figure 1D).

\section{Fourier-transform infrared spectroscopy}

The origins of the bonding between the silver nanoparticle surface and the capping molecule were investigated by FTIR spectroscopy. In the FTIR analysis, PGA was used as a comparison sample. The infrared spectra of the PGA and PGA-capped silver nanoparticles in the $\mathrm{KBr}$ pellet showed strong characteristic $\mathrm{C}-\mathrm{N}$ stretching at about 1000 $1150 \mathrm{~cm}^{-1}$, carbonyl absorption at about $1390-1420 \mathrm{~cm}^{-1}$, and strong hydroxyl absorption at $3200-3550 \mathrm{~cm}^{-1} \cdot{ }^{36}$ The absorption peaks at $3380-3410 \mathrm{~cm}^{-1}$ were indicative of $\mathrm{N}-\mathrm{H}$ stretching. ${ }^{37,38}$ The absorption peaks at around $1600-1690 \mathrm{~cm}^{-1}$ and $1395-1400 \mathrm{~cm}^{-1}$ were characteristic of amide groups and $\mathrm{C}=\mathrm{O}$ groups. Glutamic acid, the building monomer of polyglutamic acid, possesses three chemically active functional groups, ie, $\alpha-\mathrm{NH}_{2}, \alpha-\mathrm{COOH}$, and $\gamma-\mathrm{COOH}$. The chemical reactivity of these three functional groups follows the order $\alpha-\mathrm{NH}_{2}>\alpha-\mathrm{COOH},>\gamma-\mathrm{COOH} .{ }^{41}$ The FTIR spectra for PGA and the PGA-capped silver nanoparticles confirmed the presence of carboxyl, hydroxyl, carbonyl, and amide groups.

Comparing the FTIR spectra obtained for PGA (0.1)capped silver nanoparticles and PGA (0.4)-capped silver nanoparticles with the characteristic FTIR spectra of PGA, it is reasonable to believe that the silver nanoparticles obtained were capped with PGA. Details of the spectra interpretation and band assignments are shown in Table 1.

\section{Field emission scanning electron microscopy}

The morphological characteristics of the bare silver nanoparticles and those capped with different amounts of PGA were observed by FESEM. The FESEM images show the crucial influence of the amount of capping agent on the morphology of silver nanoparticles. Figure 2A shows the FESEM micrographs recorded for the bare silver nanoparticles, from which one can see that the particles are much agglomerated. The bare silver nanoparticles have been joined together and assembled, forming clusters, fibers, and a rope-like structure. The main reason for this phenomenon is lack of use of a stabilizer in the experiment.

Figure 2B shows the FESEM images for the microstructure of silver nanoparticles capped with $0.4 \%$ of PGA. These particles are much agglomerated and have formed a film, on which cracks and roughness can be seen, and sporadically distributed spherical particles of smaller and larger sizes are still present. From these results, it is obvious that the concentration of capping agent must be optimized in order to obtain the smallest particle dimensions using this method, as well as to keep agglomeration to a minimum. A $0.4 \%$ PGA concentration is clearly too high for this method.

The micrograph for silver nanoparticles capped with $0.1 \%$ of PGA shows that the particles have a nearly spherical shape, are smooth-surfaced, and have a low level of agglomeration and a high level of uniformity (Figure 3). The size distribution of all nanoparticles was unimodal, with particle sizes of about 5-45 nm.

\section{Transmission electron microscopy}

The silver nanoparticles obtained at various capping agent/ silver ratios were also examined by TEM. Silver nanoparticles obtained in the experiment without PGA showed irregular morphology with a broad size distribution. From Figure 4A it can be seen that lack of a stabilizer in the system leads to formation of agglomerates. Further analyses of the

Table I Assignment, vibrational modes, and positions (wave number in $\mathrm{cm}^{-1}$ ) of FTIR bands in the spectra of PGA, AgNpPGA (0.I), and AgNpPGA (0.4)

\begin{tabular}{llllll}
\hline Assignment & Vibrational modes & \multicolumn{2}{l}{ Band position (cm $\left.{ }^{-1}\right)$} & Appearance \\
\cline { 3 - 5 } & & PGA & AgNpPGA (0.1) & AgNpPGA (0.4) & Broad and strong \\
\hline O-H & Stretching & 3380 & 3410 & 3400 & Medium to strong \\
Amide I & Associated amides & 1650 & 1600 & 1630 & Medium to strong \\
$\mathrm{C}=\mathrm{O}$ & Symmetric & 1400 & 1400 & 1390 & Weak to medium \\
$\mathrm{N}-\mathrm{H}$ & $(\delta)$ In-plane bending or scissoring & 1300 & 1300 & 1300 & Broad and strong \\
$\mathrm{C}-\mathrm{N}$ & Stretching & 1050 & 1080 & 1150 & Weak \\
$\mathrm{N}-\mathrm{H}$ & $(\omega)$ Out-of-plane bending or wagging & 760 & 730 & 750 & \\
\hline
\end{tabular}

Abbreviations: FTIR, Fourier transform infrared spectroscopy; PGA, poly- $\alpha, \gamma$, L-glutamic acid; AgNpPGA, PGA-capped silver nanoparticles. 

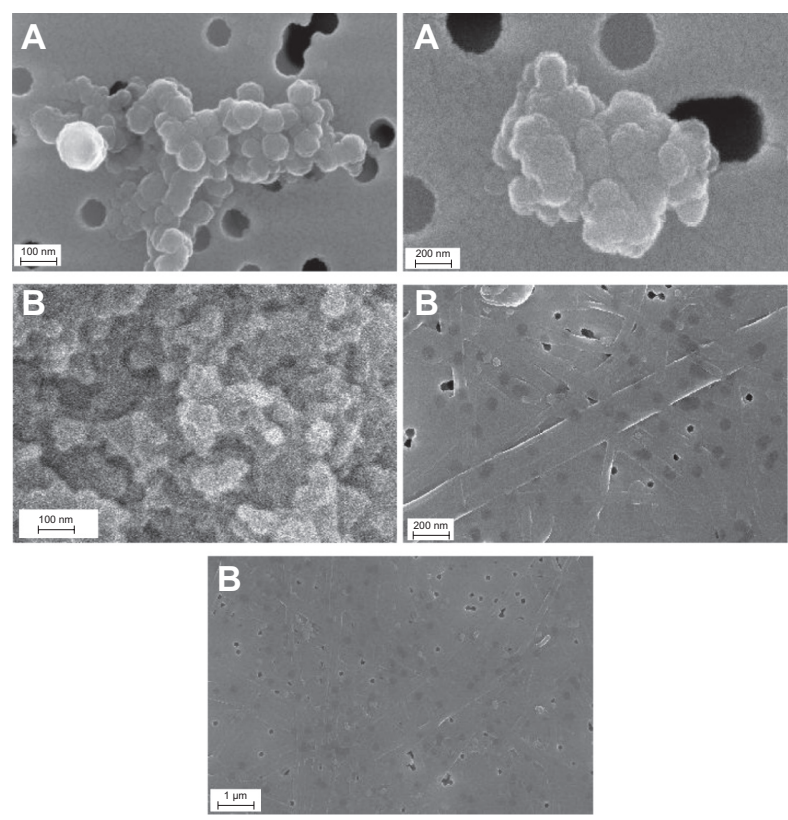

Figure 2 Images of $\mathrm{AgNp}$ particles obtained in the experiment without PGA (A) and with $0.4 \%$ PGA (B).

Abbreviations: FESEM, field emission scanning electron microscope; PGA, poly- $\alpha$, $\gamma$, L-glutamic acid

0.1\% PGA-capped silver nanoparticles using TEM showed nearly spherical nanoparticles with diameters in the range of 5-45 nm. From Figure 4B, it is clear that the particles had a low level of agglomeration and a high level of uniformity. TEM images of silver nanoparticles capped with
$0.4 \%$ PGA confirm the majority of the previously mentioned considerations. These nanoparticles have irregular shapes and are very much agglomerated (Figure 4C). Both the FESEM and TEM images demonstrate that the use of different amounts of PGA leads to nanoparticles with significantly different morphologies.

The proposed mechanism for formation of colloidal PGA-capped silver nanoparticles is shown in Figure 5. The observations reported here suggest that formation of PGAcapped silver nanoparticles from $\mathrm{AgNO}_{3}$ comprises several steps. In the first step, silver ions are reduced, leading to formation of silver clusters. In the second step, absorption of PGA onto the surface of silver clusters, further reduction of adjacent silver ions, and their accumulation on silver clusters occurs, followed by growth of the silver particles and colloidal stabilization by PGA. Flexible linkage of PGA and the large number of molecules in the reaction solution leads to isotropic growth and the formation of stable spherical silver nanoparticles. With further increases in PGA concentration, large-sized silver nanoparticles and agglomerates are formed. As a consequence, in a colloidal suspension of given concentration, excessive concentration of polymers leads to an increasing number of bridges (polymers interconnecting different aggregates), and finally to the formation of a three-dimensional polymer network (see Figures 2B, $4 \mathrm{C}$, and 5$).{ }^{38}$
A
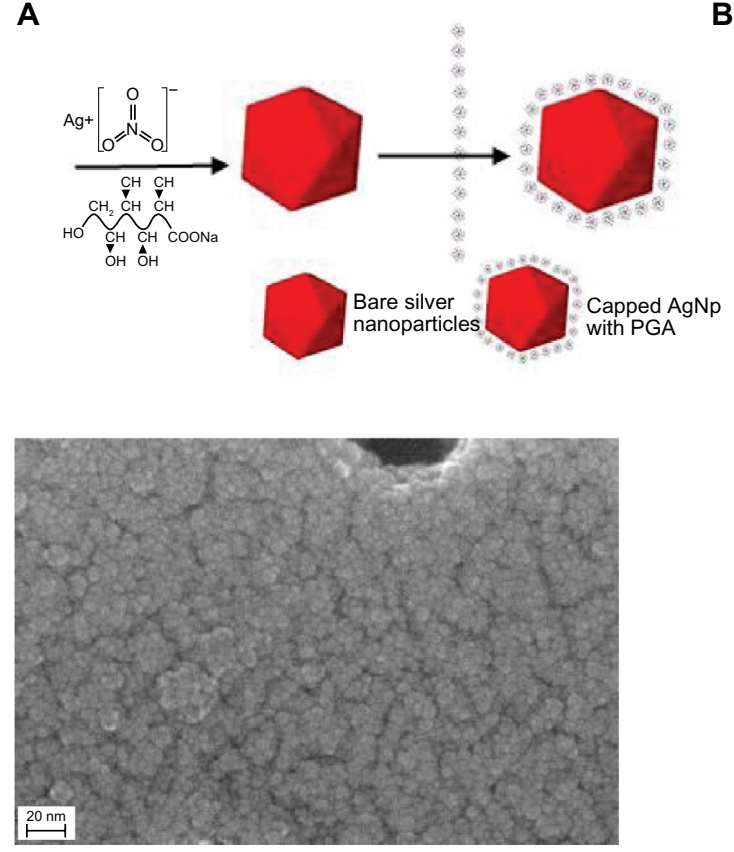

B
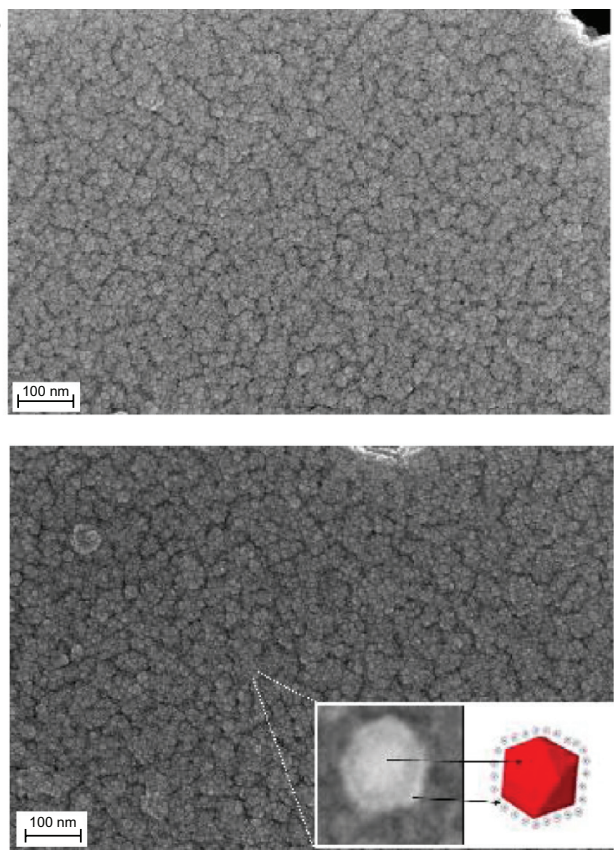

Figure 3 (A) Scheme of the green synthesis of the bare and capped silver nanoparticles. (B) FESEM images of capped silver nanoparticles with $0.1 \%$ PGA (inset shows arbitrarily magnified particle).

Abbreviations: AgNp, silver nanoparticles; FESEM, Field Emission Scanning Electron Microscope; PGA, PGA, poly- $\alpha, \gamma$, L-glutamic acid. 
A

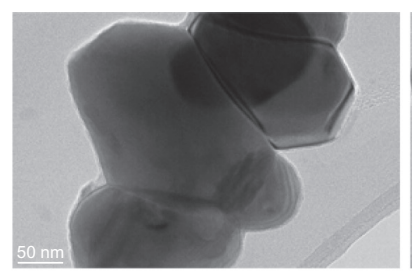

B

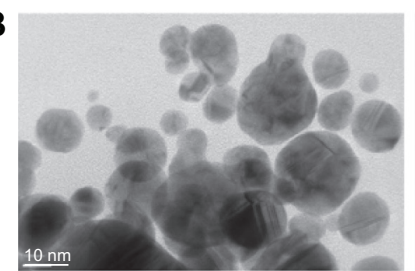

C

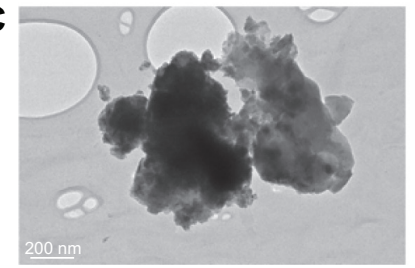

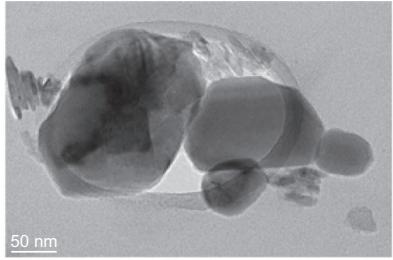
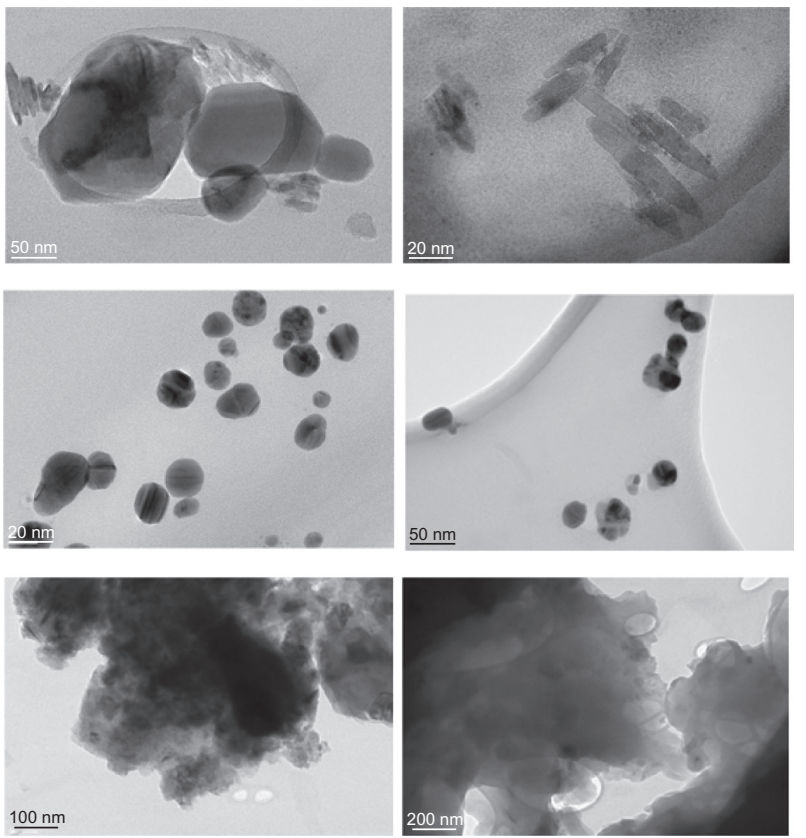

Figure 4 TEM images of bare silver nanoparticles (A), capped with $0.1 \%$ PGA (B) and with $0.4 \%$ PGA (C) Abbreviations: TEM, transmission electron microscope; PGA, poly- $\alpha, \gamma$, L-glutamic acid.

\section{Zeta potential}

The results of zeta potential determination for the bare and PGA-capped silver nanoparticles are shown in Table 2. The zeta potential was reported as the average of five readings taken per sample, including the standard deviation. The zeta potential is directly proportional to the stability of a colloid. If all the particles in a colloid have a large negative or positive zeta potential, they will tend to repel each other and there will be a low likelihood of the particles coming together. PGA was used as a stabilizer, creating negatively charged silver particles with a specific zeta potential.

The particle size was lower for silver nanoparticles stabilized and capped with $0.05 \%$ and $0.1 \%$ PGA compared with
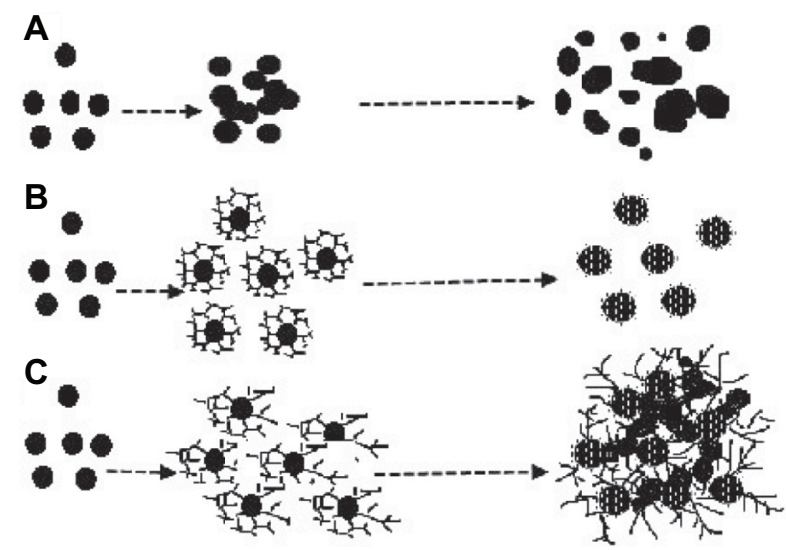

Figure 5 Scheme representing proposed formation of colloidal silver nanoparticles without PGA (A) with optimal (B) and excessive (C) concentration of PGA. Abbreviation: PGA, poly- $\alpha, \gamma$, L-glutamic acid. the bare nanoparticles. Although the PGA layer contributes to the size of the particles, this serves as evidence of reduction in particle size, aiding precipitation of the silver nanoparticles, presumably by adsorbing onto the growing particle facets. However, this is not the case for particles capped with $0.4 \%$ PGA. The polydispersity index, which is a dimensionless number indicating the width of a size distribution and having a value between 0 and 1 , was also determined. Narrow dispersions have polydispersity index values $<0.2$, broader dispersions have values of $0.2-0.5$, and very dispersed particles have values $>0.5 .^{39,40}$ According to Table 2, dispersions of PGA (0.05)-capped and (0.1)-capped silver nanoparticles had polydispersity index values slightly higher than 0.2 , which is in good agreement with the results obtained by FESEM and TEM. It is also shown in Table 2 that PGA (0.4)-capped silver nanoparticles are widely dispersed particles.

Zeta potentials for all the samples, except for PGA (0.4)-capped silver nanoparticles, are higher than $-30 \mathrm{mV}$, indicating good stability of this colloidal system. ${ }^{41}$ Electrophoretic mobility is inversely proportional to the viscosity of the medium, and viscosity is directly proportional to PGA concentration, so electrophoretic mobility decreases with increasing concentrations of PGA.$^{42}$ The zeta potential is directly proportional to electrophoretic mobility ${ }^{42}$ (according to the Henry equation: $U_{E}=2 \varepsilon z f(k a) / 3 \eta$, where $\mathrm{U}_{\mathrm{E}}=$ electrophoretic mobility, $\varepsilon=$ the dielectric constant, $\mathrm{z}=$ the zeta potential, $\eta=$ viscosity and $\mathrm{f}(\mathrm{ka})=$ Henry's function), and both the zeta potential and colloidal stability 
Table 2 Zeta potential results for bare and PGA-capped silver nanoparticles

\begin{tabular}{lclll}
\hline Sample & Particle size $(\mathbf{n m})$ & pH & PDI & Zeta potential (mV) \\
\hline AgNp & $69.2 \pm 5.0$ & $4.30-4.37$ & 0.235 & $-38.5 \pm 11.5$ \\
AgNpPGA (0.05) & $47.5 \pm 5.0$ & & 0.201 & $-49.1 \pm 19.0$ \\
AgNpPGA (0.1) & $44.9 \pm 5.0$ & & 0.206 & $-43.7 \pm 12.0$ \\
AgNpPGA (0.4) & $179.0 \pm 5.0$ & 0.707 & $-10.8 \pm 3.8$
\end{tabular}

Note: Values are mean \pm standard deviation $(n=5)$.

Abbreviations: PDI, polydispersity index; PGA, poly- $\alpha, \gamma$, L-glutamic acid; AgNpPGA, PGA-capped silver nanoparticles.

decreased with increasing PGA concentrations, which is in good agreement with the ultraviolet-visible results (Figure 1D).

\section{In vitro cytotoxicity of bare and PGA-capped silver nanoparticles}

The cytotoxicity of the bare and PGA (0.1)-capped silver nanoparticles was determined by MTT assay after exposure of HepG2 cells to $0 \%, 0.01 \%, 0.1 \%, 1 \%$, and $10 \% \mathrm{v} / \mathrm{v}$ of silver nanoparticles and PGA (0.1)-capped silver nanoparticles for 24 hours. Cell viability data for the vehicle control are not shown in Figure 6 because they were almost identical to the negative control. At concentrations up to $1 \%(\mathrm{v} / \mathrm{v})$, both types of particles exerted a similar effect on the viability of HepG2 cells. At these concentrations, the viability was not reduced by more than $20 \%$ compared with nontreated controls. At $10 \%(\mathrm{v} / \mathrm{v})$, the silver nanoparticles reduced cell viability by $35 \%$ compared with the control, while the viability of cells exposed to $10 \%(\mathrm{v} / \mathrm{v})$ PGA (0.1)-capped silver nanoparticles was reduced by $17 \%$ (Figure 6 ).

\section{Induction of intracellular reactive oxygen species}

To establish the influence of bare and PGA (0.1)-capped silver nanoparticles on intracellular reactive oxygen species

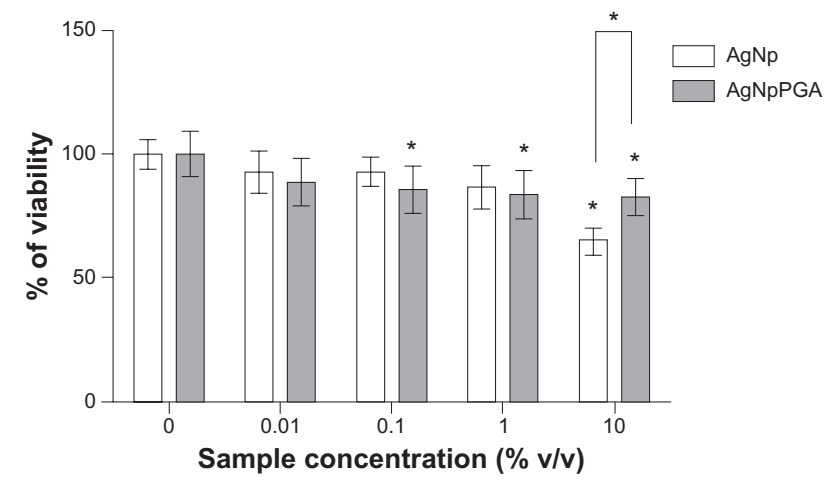

Figure 6 Viability of HepG2 cells treated with AgNp or AgNpPGA (0.1) for $24 \mathrm{~h}$. The data are presented as mean values of three independent experiments (each with five replicates) $\pm S D$.

Note: $* P<0.05$ statistically significant difference (ANOVA, Kruskal-Wallis). Abbreviations: AgNp, silver nanoparticles; AgNpPGA, PGA-capped silver nanoparticles; SD, standard deviation; PGA, poly- $\alpha, \gamma$, L-glutamic acid. formation, we measured the kinetics of their formation in HepG2 cells by DCFH-DA assay. Reactive oxygen species induction data for the vehicle control are not shown in Figure 7, because they were almost identical to those of the negative control.

Both samples induced a significant increase in formation of intracellular reactive oxygen species, but only at the highest tested concentration $(10 \% \mathrm{v} / \mathrm{v})$, whereas the bare silver nanoparticles had a stronger influence on formation of reactive oxygen species compared with PGA (0.1)-capped silver nanoparticles. Reactive oxygen species formation increased by 2.2-fold after five hours of exposure to the bare silver nanoparticles and was 1.8-fold higher than in control cells after exposure to PGA (0.1)-capped silver nanoparticles (Figure 7).

\section{Conclusion}

In conclusion, bare and PGA-capped silver nanoparticles were successfully synthesized using a new, simple, green, one-pot, four-component method. The cytotoxic potential and influence of these nanoparticles on formation of intracellular reactive oxygen species was investigated in vitro. A naturally occurring anionic polymer was used as the organic layer to protect the silver nanoparticles from agglomeration and render them biocompatible, and glucose was used as a reducing agent. One of the advantages of this method is that ammonia (a caustic and hazardous chemical) is not used during synthesis. This method represents an attractive, rapid, and low-cost process with the potential for industrial scale-up. However, appropriate optimization is necessary to be able to develop silver nanoparticles with a narrow size distribution. Observations using ultraviolet-visible spectroscopy, FESEM, TEM, and zeta potential measurement indicate that these silver nanoparticles agglomerate if PGA is not used during synthesis and if there is an excessive concentration of PGA. The capped silver nanoparticles obtained in this experiment using the optimum concentration of PGA $0.1 \%$ were uniform and nonagglomerated, had a narrow particle size distribution, and were highly stable for several months. This PGA concentration should be used for manufacture of PGA-capped silver nanoparticles when scaled up to large batches. The MTT assay 
A

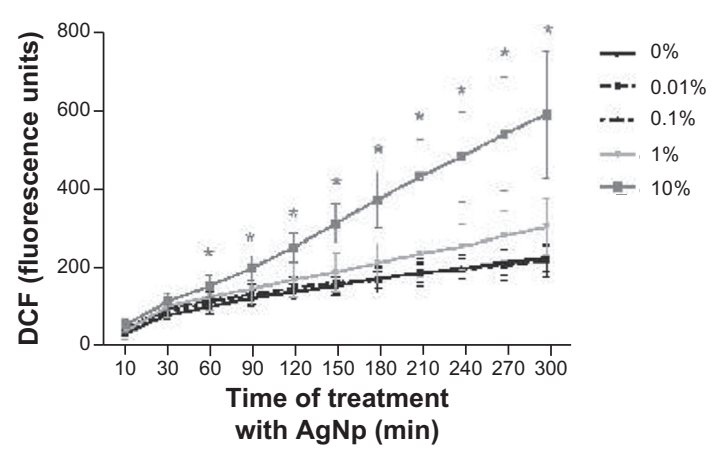

B

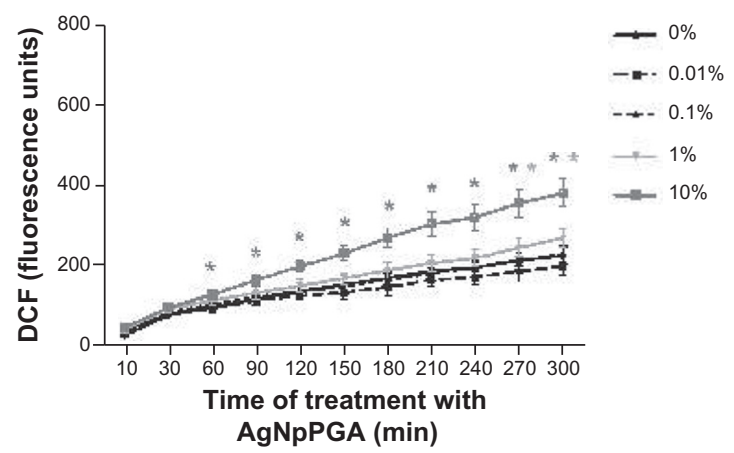

C

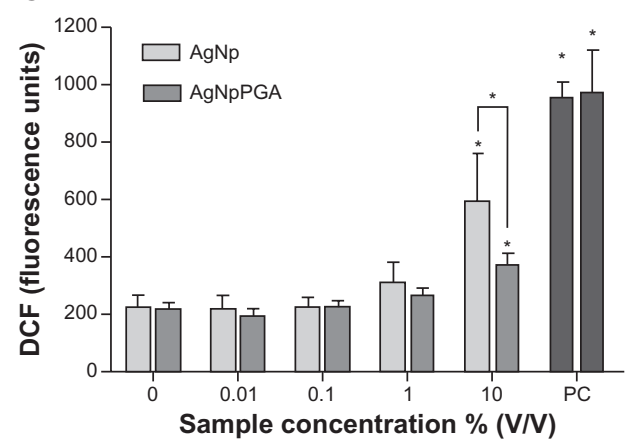

Figure $7 \mathrm{AgNp}$ and $\mathrm{AgNpPGA}(0.1)$-induced intracellular ROS formation in HepG2 cells. Kinetic of ROS formation during 5 hour exposure to AgNp or AgNpPGA (0.1) $(\mathbf{A}$ and $\mathbf{B})$; each point represents the mean of five replicates $( \pm S D)$ of representative experiment. Increase of DCFH fluorescence intensity in cells exposed to AgNp or $\mathrm{AgNpPGA}(0 . \mathrm{I})$ after 5 hour exposure $(\mathbf{C})$; each bar represent means $( \pm S D)$ of three independent experiments. $\mathrm{PC}(0.5 \mathrm{mM} t$-BOOH).

Note: $* P<0.05$; statistically significant difference (ANOVA, Kruskal-Wallis).

Abbreviations: DCF, 2-7-dichlorofluorescin; PGA, poly- $\alpha, \gamma$, L-glutamic acid; AgNp, silver nanoparticles; AgNpPGA, PGA-capped silver nanoparticles; ROS, reactive oxygen species; DCFH, 2-7-dichlorofluorescin; SD, standard deviation; PC, positive control.

results indicated that PGA (0.1)-capped silver nanoparticles have good biocompatibility, and do not increase the production of reactive oxygen species by HepG2 cells in vitro.

\section{Acknowledgment}

This study was supported by a grant from the Ministry of Science and Technological Development of the Republic of Serbia (Grant No. III45004: Molecular designing of nanoparticles with controlled morphological and physicochemical characteristics and functional materials based on them). The authors would like to thank Srečo Škapin and Ines Bračko for field emission scanning electron microscopy and TEM measurements.

\section{Disclosure}

The authors report no conflicts of interest in this work.

\section{References}

1. Dahl A, Maddux BL, Hutchison JE. Toward greener nanosynthesis. Chem Rev. 2007;107(6):2228-2269.

2. Sau TK, Rogach AL, Jäckel F, Klar TA, Feldmann J. Properties and applications of colloidal nonspherical noble metal nanoparticles. $A d v$ Mat. 2010;22(16):1805-1825.
3. Sönnichsen C, Reinhard BM, Liphardt J, Alivisatos AP. A molecular ruler based on plasmon coupling of single gold and silver nanoparticles. Nat Biotechnol. 2005;23(6):741-745.

4. McEachran M, Keogh D, Pietrobon B, et al. Ultrathin gold nanoframes through surfactant-free templating of faceted pentagonal silver nanoparticles. J Am Chem Soc. 2011;133(21):8066-8069.

5. Barud HS, Regiani T, Marques RFC, Lustri WR, Messaddeq Y, Ribeiro SJL. Antimicrobial bacterial cellulose-silver nanoparticles composite membranes. J Nanomater. 2011.

6. Sharma VK, Yngard RA, Lin Y. Silver nanoparticles: green synthesis and their antimicrobial activities. Adv Colloid Interface Sci. 2009; 145(1-2):83-96.

7. Shon YS, Cutler E. Aqueous synthesis of alkanethiolate-protected Ag nanoparticles using Bunte salts. Langmuir. 2004;20(16):6626-6630.

8. Chen H, Liu Y, Zhao G. Synthesis and characterization of hollow silver spheres at room temperature. Electronic Materials Letters. 2011; 7(2):151-154.

9. Park S, Murthy PS, Park S, Mohan YM, Koh WG. Preparation of silver nanoparticle-containing semi-interpenetrating network hydrogels composed of pluronic and poly(acrylamide) with antibacterial property. Ind Eng Chem. 2011;17(2):293-297.

10. Huynh KA, Chen KL. Aggregation kinetics of citrate and polyvinylpyrrolidone coated silver nanoparticles in monovalent and divalent electrolyte solutions. Environ Sci Technol. 2011;45(13):5564-5571.

11. Park KH, Im SH, Park OO. The size control of silver nanocrystals with different polyols and its application to low-reflection coating materials. Nanotechnology. 2011;22(4):045602.

12. Shameli K, Ahmad MB, Yunus WM, et al. Green synthesis of silver/ montmorillonite/chitosan bionanocomposites using the UV irradiation method and evaluation of antibacterial activity. Int J Nanomedicine. 2010;5:875-887. 
13. Shameli K, Ahmad MB, Zargar M, Yunus WM, Ibrahim NA. Fabrication of silver nanoparticles doped in the zeolite framework and antibacterial activity. Int J Nanomedicine. 2011;6:331-341.

14. Mahendia S, Tomar AK, Chahal RP, Kumar S. Synthesis and characterization of silver-poly(vinyl alcohol) nanocomposites. Solid State Physics. 2011;1349:339-340.

15. Cinar S, Gündül G, Mavis B, Colak U. Synthesis of silver nanoparticles by oleylamine-oleic acid reduction and its use in making nanocable by coaxial electrospinning. J Nanosci Nanotechnol. 2011; 11(4):3669-3679.

16. Dongwei W, Weiping Q. Facile synthesis of Ag and Au nanoparticles utilizing chitosan as a mediator agent. Colloids Surf B Biointerfaces. 2008;62(1):136-142.

17. Darroudi M, Ahmad MB, Abdullah AH, Ibrahim NA. Green synthesis and characterization of gelatin-based and sugar-reduced silver nanoparticles. Int J Nanomedicine. 2011;6:569-574.

18. Raveendran P, Fu J, Wallen SL. Completely "green" synthesis and stabilization of metal nanoparticles. J Am Chem Soc. 2003;125(46) 13940-13941.

19. Stevanović M, Radulović A, Jordović B, Uskoković D. Poly(DLlactide-co-glycolide) nanospheres for the sustained release of folic acid. J Biomed Nanotechnol. 2008;4(3):349-358.

20. Guidelli EJ, Ramos AP, Zaniquelli ME, Baffa O. Green synthesis of colloidal silver nanoparticles using natural rubber latex extracted from Hevea brasiliensis. Spectrochim Acta A Mol Biomol Spectrosc. 2011; 82(1):140-145.

21. Shih IL, Van YT. The production of poly $(\gamma$-glutamic acid) from microorganisms and its various applications. Bioresour Technol. 2001 79(3):207-225.

22. Yu DG. Formation of colloidal silver nanoparticles stabilized by Na+poly(gamma-glutamic acid)-silver nitrate complex via chemical reduction process. Colloids Surf B Biointerfaces. 2007;59(2):171-178.

23. Sarkar P, Pyne S, Sahoo GP, et al. Solution-phase synthesis of silver nanodiscs in HPMC-matrix and simulation of UV-vis extinction spectra using DDA based method. Spectrochim Acta A Mol Biomol Spectrosc. 2011;82(1):368-374.

24. El-Shishtawy RM, Asiri AM, Al-Otaibi MM. Synthesis and spectroscopic studies of stable aqueous dispersion of silver nanoparticles. Spectrochim Acta A Mol Biomol Spectrosc. 2011;79(5):1505-1510.

25. Tolaymat TM, El Badawy AM, Genaidy A, Scheckel KG, Luxton TP, Suidan M. An evidence-based environmental perspective of manufactured silver nanoparticle in syntheses and applications: a systematic review and critical appraisal of peer-reviewed scientific papers. Sci Total Environ. 2010;408(5):999-1006.

26. Christensen FM, Johnston HJ, Stone V, et al. Nano-silver-feasibility and challenges for human health risk assessment based on open literature. Nanotoxicology. 2010;4(3):284-295.
27. Kim S, Choi JE, Choi J, et al. Oxidative stress-dependent toxicity of silver nanoparticles in human hepatoma cells. Toxicol In Vitro. 2009;23(6):1076-1084.

28. Schins RP, Knaapen AM. Genotoxicity of poorly soluble particles. Inhal Toxicol. 2007;19 Suppl 1:189-198.

29. Mosmann T. Rapid colorimetric assay for cellular growth and survival: application to proliferation and cytotoxic assays. J Immunol Methods. 1983;65(1-2):55-63.

30. Stevanović M, Maksin T, Petković J, Filipič M, Uskoković D. An innovative, quick and convenient labeling method for the investigation of pharmacological behavior and the metabolism of poly(DL-lactideco-glycolide) nanospheres. Nanotechnology. 2009;20(33):335102.

31. Osseni RA, Debbasch C, Christen MO, Rat P, Warnet JM. Tacrineinduced reactive oxygen species in a human liver cell line: the role of anethole dithiolethione as a scavenger. Toxicol In Vitro. 1999;13(4-5): 683-688.

32. Petković J, Žegura B, Stevanović M, et al. DNA damage and alterations in expression of DNA damage responsive genes induced by $\mathrm{TiO}(2)$ nanoparticles in human hepatoma HepG2 cells. Nanotoxicology. 2010; 5:341-353.

33. Sönnichsen C, Franzl T, Wilk T, von Plessen G, Feldmann J. Plasmon resonances in large noble-metal clusters. New J Phys. 2002;4(1):93.

34. Heath JR. Size-dependent surface-plasmon resonances of bare silver particles. Phys Rev B Condens Matter. 1989;40(14):9982-9985.

35. Hao E, Schatz GC. Electromagnetic fields around silver nanoparticles and dimmers. J Chem Phys. 2004;120(1):357-366.

36. Dong TY, Chen WT, Wang CW, et al. One-step synthesis of uniform silver nanoparticles capped by saturated decanoate: direct spray printing ink to form metallic silver films. Phys Chem Chem Phys. 2009;11(29):6269-6275.

37. Ho GH, Ho TI, Hsieh KH, et al. $\gamma$-Polyglutamic acid produced by bacillus subtilis (natto): structural characteristics, chemical properties and biological functionalities. J Chinese Chem Soc. 2006;53:1363-1384.

38. Meier W, Hotz J, Gunther-Ausborn S. Vesicle and cell networks: interconnecting cells by synthetic polymers. Langmuir. 1996;12: 5028-5032.

39. Wissing SA, Kayser O, Muller RH. Solid lipid nanoparticles for parenteral drug delivery. Adv Drug Deliv Rev. 2004;56(9):1257-1272.

40. Villalobos-Hernández JR, Müller-Goymann CC. Novel nanoparticulate carrier system based on carnauba wax and decyl oleate for the dispersion of inorganic sunscreens in aqueous media. Eur J Pharm Biopharm. 2005;60(1):113-122.

41. Li LC, Tian Y. Zeta potential. In: Swarbrick J, Boylan JC, eds. Encyclopedia of pharmaceutical technology. 1st ed. New York: Marcel Dekker Inc; 1997:429-458.

42. Shaw DJ. Introduction to Colloid and Surface Chemistry. 4th ed. Oxford Butterworth-Heinemann; 1992.
International Journal of Nanomedicine

\section{Publish your work in this journal}

The International Journal of Nanomedicine is an international, peerreviewed journal focusing on the application of nanotechnology in diagnostics, therapeutics, and drug delivery systems throughout the biomedical field. This journal is indexed on PubMed Central,

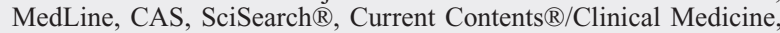

\section{Dovepress}

Journal Citation Reports/Science Edition, EMBase, Scopus and the Elsevier Bibliographic databases. The manuscript management system is completely online and includes a very quick and fair peer-review system, which is all easy to use. Visit http://www.dovepress.com/ testimonials.php to read real quotes from published authors. 